\title{
Map-Based Music Interfaces for Mobile Devices
}

\author{
Jakob Frank, Thomas Lidy, Peter Hlavac and Andreas Rauber \\ Vienna University of Technology, Department of Software Technology and Interactive Systems \\ Favoritenstraße 9-11/188 \\ Vienna, Austria \\ \{frank,lidy,rauber\}@ifs.tuwien.ac.at, peter.hlavac@ researchstudio.at
}

\begin{abstract}
The pervasion of digital music calls for novel techniques to search, retrieve and access music collections. Particularly mobile devices are, due to their limited display size and input capabilities, in a need of possibilities for intuitive and quick selection of music that go beyond mere browsing through song lists and directories. We propose a graphical user interface for mobile devices presenting a music map that organizes a music collection automatically by sound similarity through audio analysis. This map provides an overview over large audio collections and offers several interaction possibilities to give users a quick and direct access to their music. It allows instant creation of playlists based on music of a desired genre by pointing on clusters or drawing paths on the map. The application not only eases access to music, but also enables novel application scenarios for collaborative music experience. The software has been implemented for a range of mobile devices, such as PDAs and smartphones.
\end{abstract}

Categories and Subject Descriptors: H.5.2 [Information Interfaces and Presentation]: User Interfaces

General Terms: Design, Human Factors.

\section{INTRODUCTION}

Through the pervasion of $3 \mathrm{G}$ networks and the mobile Internet the market for consuming music on mobile devices has evolved and will soon become a major distribution channel. Increasing bandwidth and lower data transfer costs encourage users to access digital content over their mobile phones. First flat rate services already exist, where customers can stream or download music and reproduce their songs without limitations as long as the monthly subscription is maintained. Consuming music on mobile devices, however, is often less convenient because of limited capabilites compared to desktop systems. Displays are much smaller and the lack of a full keyboard makes it difficult to select a set of songs. Thus, quick and intuitive access to the music itself is a key requirement on mobile devices.

We present PocketSOM, a graphical user interface provid-

Copyright is held by the author/owner(s).

MM'08, October 26-31, 2008, Vancouver, British Columbia, Canada. ACM 978-1-60558-303-7/08/10. ing navigation opportunities for quick and intuitive access to music on mobile devices. The software visualizes automatically organized music collections on a music map and gives the possibility to easily create playlists by pointing at a cluster of music of a specific style or drawing a trajectory over the map. Playlists generated in this manner can be played from the device, streamed from the Internet or sent to an external player. We implemented several prototypes on different mobile platforms and will demonstrate these new forms of exploring and interacting with music libraries and also present scenarios for novel music experience.

\section{TECHNICAL BACKGROUND}

The music map approach as an interface to music collections originated in the SOM-enhanced JukeBox (SOMeJB) project [5]. Music maps are created by (1) applying audio analysis and feature extraction methods, (2) using the Self-Organizing Map algorithm for clustering and organization of the music collection and (3) applying a visualization technique to achieve an "Islands of Music"-like visualization. More specifically, the frequency spectrum of the music is analyzed according to human perception, including psycho-acoustic models. The resulting sets of features, such as Rhythm Patterns, Rhythm Histograms and Spectrum Descriptors [2], provide a description of the content of the music that is used as input to a Self-Organizing Map (SOM) [1]. A SOM maps the high-dimensional feature data to a two-dimensional map grid while preserving relationships in the input data. The result is a map of music, containing an entire music collection, where acoustic similarity between neighbouring pieces of music is maintained. This spatial arrangement provides users an overview and direct access to a collection. It allows to intuitively explore the collection and quickly locate similar music around known pieces. Different visualizations of a map have been devised, an important one for visualizing clusters is Islands of Music, relying on Smoothed Data Histograms [5].

PocketSOM relies on the PlaySOM desktop application for the creation of music maps. In [2] the PocketSOMPlayer for the iPAQ PocketPC was presented, the first prototype of the music map paradigm for a mobile device. PocketSOM has meanwhile been extended to other mobile platforms and incorporates now a number of novel features for interaction, as described below. Web services for feature extraction as well as SOM training are available for creating maps of personal music collections. ${ }^{1}$

\footnotetext{
${ }^{1}$ http://www.ifs.tuwien.ac.at/mir/webservice/
} 


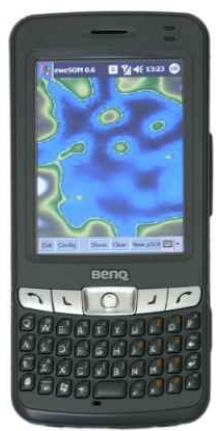

(a) BenQ P50

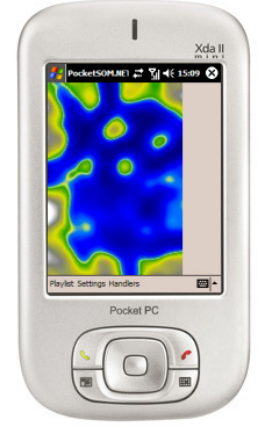

(b) HTC Magican

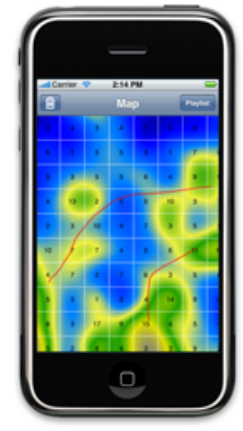

(c) iPhone
Figure 1: PocketSOM on selected devices.

\section{POCKETSOM}

PocketSOM ${ }^{2}$ presents a music map, giving a quick overview over large audio collections on devices with limited interaction possibilities. It helps navigating through the collection and provides an intuitive way of creating playlists [4]. We developed 5 different and independent implementations, each based on a different platform, as described in the following paragraphs. Three of the prototypes are visible in Figure 1.

iPocketSOM was the first prototype implementation of PocketSOM. It is based on Java and SWT, and was developed and tested on an iPAQ Pocket PC.

ePocketSOM is based on eweVM, a Java-like open source virtual machine available for a wide range of devices, e.g. PocketPC, MS SmartPhone, HandHeldPC Pro (tested on a BenQ P50 PDAphone running Windows Mobile 2003 SE).

mPocketSOM is based on JavaME and therefore runs on any mobile device that provides a touchscreen, CLDC 1.1, MIDP 2.0 and an implementation of the MMAPI that supports MP3 playback (tested on different mobile phones, including Nokia 7710 and Sony-Ericsson M600).

PocketSOM.NET is based on the .NET Compact Framework, integrating tightly with the operating system (tested on the HTC Magican).

iSOM is developed for the iPhone in XCode using Objective $\mathrm{C}$ and integrates perfectly into the iPhone environment.

While each member of the PocketSOM Family is developed for a specific group of devices, making use of special features that are only available on these platforms, there is a common set of main features offered by all PocketSOM variants: After loading the data file, containing the map and the references to the music files in the collection, the $m u$ sic map is displayed. Using the touchscreen, the user can then draw a path on the map which will result in a playlist. The songs on this playlist are selected along the trajectory drawn. Afterwards, the user can manually edit the playlist, e.g., deleting or reordering items. Finally, the playlist is further processed by one of four different output modes:

Local Playback: Playlists generated by PocketSOM can be directly played on the device with an installed audio player. The songs can be availabe locally or streamed via an Internet connection.

Exporting a Playlist File: The playlist resulting from

\footnotetext{
${ }^{2}$ http://www.ifs.tuwien.ac.at/mir/pocketsom/
}

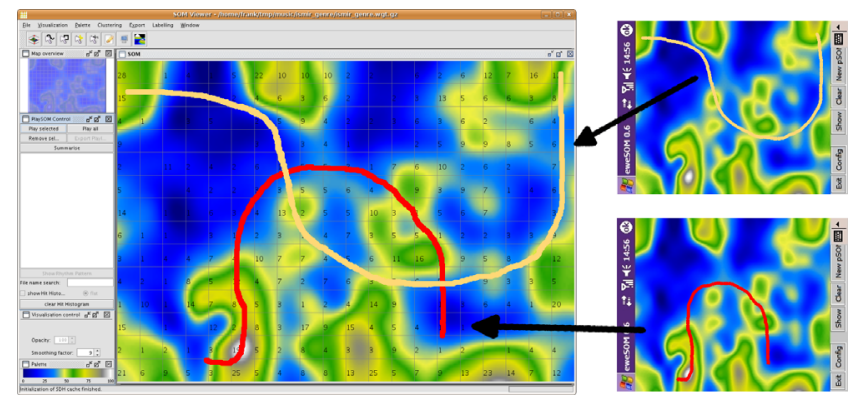

Figure 2: PocketSOM with path-sending enabled.

a path selection can be saved to an M3U file, which is then opened with a local audio player or on another device.

Remote Control allows the use of PocketSOM as a remote control by sending the playlist to a player on a remote device which takes care of playback. This player could run on the same host where the music collection is stored on (e.g. the media PC in the living room) or stream it over a network connection from another location.

Path Sending, illustrated in Figure 2, enables the mobile device to send a path drawn on the screen of the mobile device in real-time to the PlaySOM application on the desktop PC. Music selected on several devices is visualized on the desktop computer and played back on either devices, supporting cooperative creation of playlists by multiple users.

\section{CONCLUSIONS}

PocketSOM provides a graphical user interface with a music map representing a music collection. Due to the organization based on sound similarity playlists created by drawing on the map consist of similar sounding music from a selected style, with gradual changes from one genre or style to another. Numerous application scenarios were outlined, which potentially change the way we listen to music. A very interesting one, in particular facing the rising interest for social online networks, is collaborative playlist creation, where multiple users create playlists with their music map on their device, which are then fused and the listeners have the possibility to influence (or vote on) the forthcoming audio tracks.

\section{REFERENCES}

[1] T. Kohonen. Self-Organizing Maps. Springer, Berlin, 2001.

[2] T. Lidy and A. Rauber. Evaluation of feature extractors and psycho-acoustic transformations for music genre classification. Proc. ISMIR 2005, London, UK, Sep. 2005.

[3] R. Neumayer, M. Dittenbach, and A. Rauber. PlaySOM and PocketSOMPlayer: Alternative interfaces to large music collections. Proc. ISMIR 2005, London, UK, Sep. 2005.

[4] R. Neumayer, J. Frank, P. Hlavac, T. Lidy, and A. Rauber. Bringing mobile based map access to digital audio to the end user. Proc. ICIAP 200\%, Modena, Italy, Sep. 2007.

[5] E. Pampalk, A. Rauber, and D. Merkl. Content-based organization and visualization of music archives. Proc. ACM Multimedia 2002, Juan-les-Pins, France, 2002. 